\title{
A new jsPsych plugin for psychophysics, providing accurate display duration and stimulus onset asynchrony
}

\author{
Daiichiro Kuroki ${ }^{1}$ \\ Published online: 22 July 2020 \\ (C) The Psychonomic Society, Inc. 2020
}

\begin{abstract}
A JavaScript framework named 'jsPsych' developed by de Leeuw (2015) is widely used for conducting Web-based experiments, and its functionality can be enhanced by using plugins. This article introduces a new jsPsych plugin which enables experimenters to set different onset times for geometric figures, images, sounds, and moving objects, and present them synchronized with the refresh of the display. Moreover, this study evaluated the stimulus onset asynchronies (SOAs) using visual and audio stimuli. The results showed that: (i) the deviations from the intended SOAs between two visual stimuli were less than $10 \mathrm{~ms}$, (ii) the variability across browser-computer combinations was reduced compared with the no-plugin condition, and (iii) the variability of the SOAs between visual and audio stimuli was relatively large (about $50 \mathrm{~ms}$ ). This study concludes that although the use of audio stimuli is somewhat limited, the new plugin provides experimenters with useful and accurate methods for conducting psychophysical experiments online. The latest version of the plugin can be downloaded freely from https://jspsychophysics.hes.kyushu-u.ac.jp under the MIT license.
\end{abstract}

Keywords JavaScript $\cdot$ Web $\cdot$ Online experiments $\cdot$ Psychophysics

Researchers are increasingly adopting Web-browsing software, such as Microsoft Edge, Apple Safari, Google Chrome, or Mozilla Firefox, for presenting stimuli and recording responses in behavioral experiments. One of the reasons for this trend is that online/Web-based experiments allow experimenters to efficiently reach large and diverse samples for psychological research. For example, Richter and Gast (2017) recruited their participants via the online platform Prolific Academic (https://www.prolific.co) and collected data from 378 participants aged 18 to 69 . Van Steenbergen, Band, and Hommel (2015) also recruited 198 participants aged 19 to 69 via Amazon Mechanical Turk (https://www.mturk.com). These kinds of studies benefit more from online experiments than from traditional offline experiments that are conducted in a typical psychological laboratory.

Previous research has shown that the accuracy of stimulus presentation and participants' response timing in an online experiment is sufficient for replication of classical laboratory-based studies. For example, it was reported that

Daiichiro Kuroki

kurokid@kyudai.jp

1 Department of Psychology, School of Letters, Kyushu University, 744 Motooka, Nishi-ku, Fukuoka 819-0395, Japan although the reaction time in a visual search task was slightly different between Web-based and lab-based experiments, the effect of the set size and the shapes of the distributions were almost the same (Chetverikov \& Upravitelev, 2016; de Leeuw \& Motz, 2016). Pauszek, Sztybel, and Gibson (2017) also successfully replicated spatial cuing effects, one of which is that the reaction time to a target cued by arrows was faster than that to a target cued by a word like "above" or "left". Bazilinskyy and de Winter (2018) replicated the effects of stimulus onset asynchrony (SOA) between the onset of a visual stimulus and that of an auditory stimulus on reaction times. In addition, sequences of keystrokes have been evaluated with reliable timing accuracy (Pinet et al., 2017). However, it should be noted that some behavioral experiments which required a very short display duration could not be replicated (Crump, McDonnell, \& Gureckis, 2013; Semmelmann \& Weigelt, 2017). More recently, Sasaki and Yamada (2019) compared the threshold of luminance contrast measured online with that measured in a laboratory, and found that there was no significant difference between the thresholds. At present, it is generally agreed that online experiments have great potential in behavioral research (for review, see Stewart, Chandler, \& Paolacci, 2017).

Many tools, libraries, and frameworks have been developed to create programs for Web-based experiments (e.g., 
Barnhoorn, Haasnoot, Bocanegra, \& van Steenbergen, 2015; Reips \& Neuhaus, 2002; Schubert, Murteira, Collins, \& Lopes, 2013; von Bastian, Locher, \& Ruflin, 2013). This article focuses on jsPsych, a JavaScript framework developed by de Leeuw (2015). The jsPsych framework includes a number of plugins for various types of online experiments. Just by setting the parameters of the plugins, experimenters can start their research on the Internet. It is not necessary for participants to install additional plugins or applets in their own computers, and jsPsych does not depend on Flash technologies, for which Adobe has decided to end development and support.

Although jsPsych seems to be a promising JavaScript library for online studies, there are some points that remain to be improved and evaluated if experiments require time-accurate and space-flexible stimulus presentation as in psychophysical research. First, this library does not provide a convenient method for setting different onset times for each stimulus, and the SOA has not yet been adequately evaluated. Second, the visual stimulus is presented automatically at the center of the window, and there is no efficient way to present it at specific coordinates. Lastly, jsPsych does not provide an easy way to present moving objects except by using movie files. These unimplemented methods might not have been needed, because it was considered that the studies were too complicated to conduct online. However, given the increase in online research and recent improvements in the performance of hardware and software, it is expected that online psychophysical experiments will be conducted in the coming years (for review, see Woods, Velasco, Levitan, Wan, \& Spence, 2015).

The goal of this study is to develop an open-source plugin for jsPsych which enables experimenters to easily present multiple stimuli with SOAs, and evaluate the SOAs using a Black Box ToolKit, which is a device for accurately measuring presentation and response times. The actual SOAs should be identical to the intended times, but if the differences are consistent across browser-computer combinations, the onset and duration of the stimuli can be calibrated. However, if there were a large systematic bias, it would be problematic. For example, if women used the Edge browser more than men, and the stimulus duration in the Edge browser was significantly longer than that in other web browsers, the women would be presented with stimuli for longer than the men. As a result, the experiment might show a false positive based on the browserdependent difference rather than the gender difference. Therefore, it is very important to reduce the variability in stimulus presentation across browser-computer combinations. The present study evaluated how accurately multiple stimuli are presented in online studies using the new jsPsych plugin.

\section{The new jsPsych plugin for Web-based psychophysics studies}

This article introduces a new open-source jsPsych plugin called jspsych-psychophysics, which is designed to set different onset times of geometric figures, images, sounds, and moving objects, and synchronize the onsets with the refresh rate of the display. The latest version of the plugin can be downloaded freely from https://jspsychophysics.hes. kyushu-u.ac.jp under the MIT license. It is compatible with jsPsych v.6.1.0.

The downloaded files include the plugin file "jspsychpsychophysics.js" and demonstration programs. To use the plugin, the plugin file must be included using the <script $>$ tag like other jsPsych plugin files. The following sections explain how to present each type of stimulus.

\section{Presenting rectangles with SOAs in a trial}

First, all the stimuli used in the program with the jspsychpsychophysics plugin must be specified as a JavaScript object as follows:

\author{
var rect_object $1=\{$ \\ obj_type: 'rect', // means a rectangle \\ startX: $250, / /$ location in the canvas \\ startY: 360 , \\ width: $300, / /$ of the rectangle \\ height: 200 , \\ line_color: '\#ffffff', \\ fill_color: '\#fffff', \\ show_start_time: 1500 // milliseconds \\ \}
}

This code means that a white rectangle is presented at coordinates $(250,360)$ in a canvas, which is an HTML element that provides many drawing tools. The origin of the coordinate is the top left of the canvas, and the unit is the pixel. The width and height of the 
rectangle are 300 and 200 pixels, respectively. The color can be specified using the HTML color names, hexadecimal (HEX) colors, and RGB values that are often used in a general HTML file. The show_start time property is the most notable in this object, and this enables the stimulus to be presented at the intended time. In this example, a white rectangle is presented $1500 \mathrm{~ms}$ after the beginning of this trial. The experimenter can present circles, lines, and texts using a similar method.

Next, a trial object has to be specified as follows:

var trial $=\{$

type: 'psychophysics',

stimuli: [rect_object1, rect_object2, rect_object3],

canvas_width: 1000,

canvas_height: 800,

background_color: '\#008000', // of the canvas

\}

The stimuli property can include all the different objects to be presented in the trial. In this example, three rectangles are presented in a canvas whose width and height are 1000 and 800 pixels, respectively, and whose color is green (HEX: \#008000). This "trial" object must be included as a timeline property of the jsPsych.init, which is a core function of jsPsych.

\section{Presenting image and audio files}

The way to present image and audio files is almost identical to that for presenting rectangles, except that the former types of files are preloaded at the beginning of the experiment. It is very important to preload the files in order to accurately present them at the intended times. The code snippet 1 shows how to preload image and audio files, and how to present them using the jspsych-psychophysics plugin. This snippet presents the happy_face $1 . j p g$, whose size is reduced to $80 \%$ at the center of the canvas $500 \mathrm{~ms}$ after the trial begins. After another $500 \mathrm{~ms}$, the tone_1.mp3 is played.

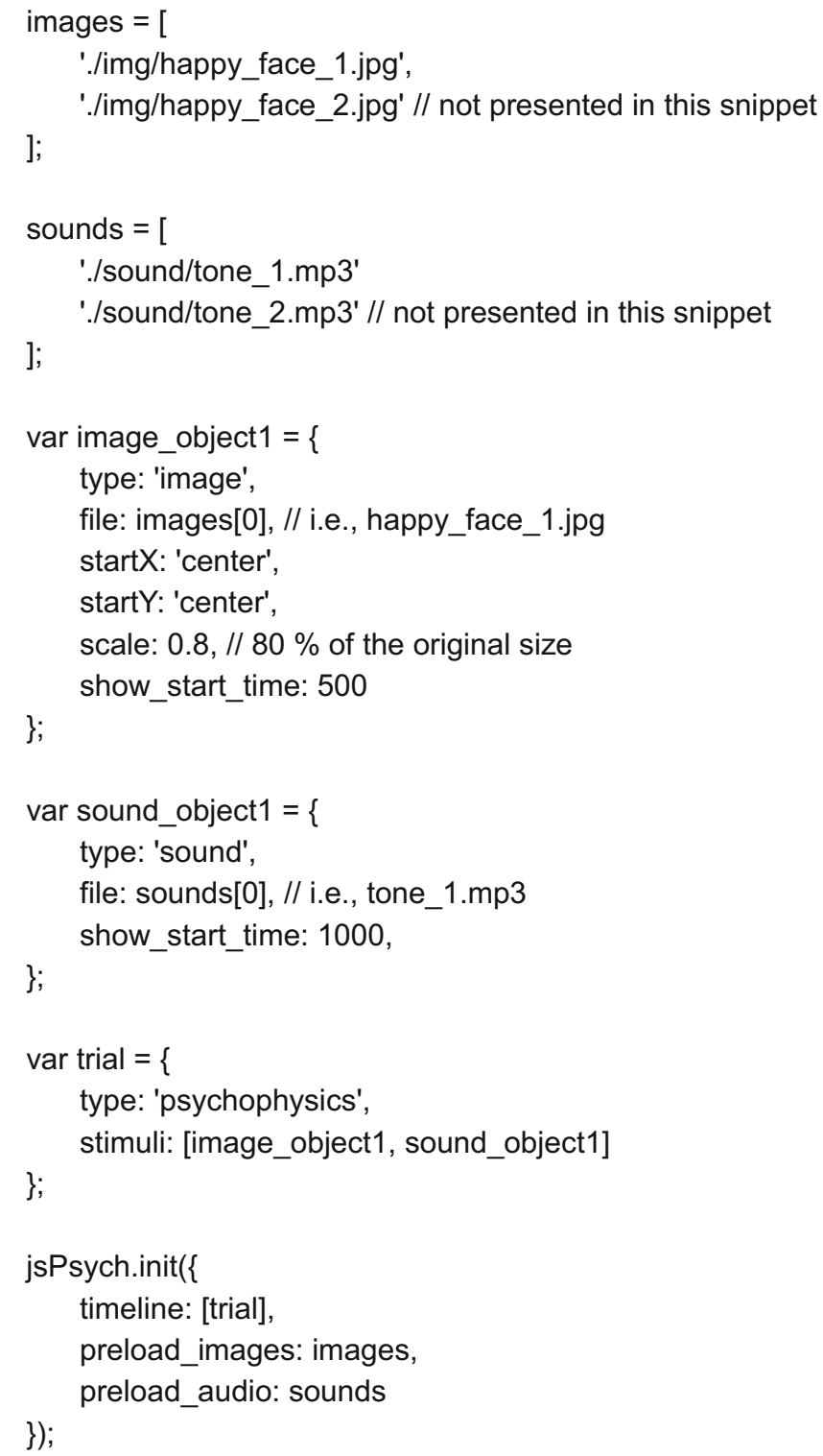

Code snippet 1. JavaScript code to preload image and audio files, and to present them using the jspsychpsychophysics plugin.

\section{Motion stimuli}

The jspsych-psychophysics plugin can change the positions of visual stimuli in sync with the refresh rate of the display using the requestAnimationFrame method. Garaizar and Reips (2019) evaluated the accuracy of the method and showed no frame loss across almost all the combinations of browsers (Chrome and Firefox) and operating systems (Windows and Linux). Moreover, Pronk, Wiers, Molenkamp, and Murre (2019) reported that the presentation time using the 
requestAnimationFrame method was more accurate than using CSS animations. To move objects using the jspsychpsychophysics plugin, the horiz_pix_sec or horiz_pix_frame property must be specified. These properties represent how far in pixels the object moves horizontally per second or frame. Similarly, the experimenter can use the vert_pix_sec and vert_pix_frame properties to move the objects vertically. Although these properties seem to work properly at first glance, evaluating the presentation time accuracy is beyond the scope of this paper. The main properties which can be used in the jspsych-psychophysics plugin are summarized in Table 1.

\section{Study 1: Evaluation of the SOAs between visual stimuli}

In the jspsych-psychophysics program, visual stimuli are presented in sync with the refresh rate of the display using the requestAnimationFrame method. Therefore, it was predicted that the presentation of the visual stimuli would be temporally more accurate than without the plugin. This study compared the accuracy with and without the plugin using the Black Box ToolKit Version 2, which is a device for psychologists, neuroscientists, and vision researchers to confirm the validity of their experimental equipment (https://www.blackboxtoolkit. com). The Black Box can detect the onset and offset of the visual and auditory stimuli using photo-sensors and microphones. Using this specialist hardware, Reimers and Stewart

Table 1 List of main properties of jspsych-psychophysics

\begin{tabular}{|c|c|}
\hline Property name & Brief explanation \\
\hline obj_type & The type of object (e.g., rectangle, image, or sound \\
\hline startX & $\begin{array}{l}\text { Location in the canvas; horizontal starting } \\
\text { position of the object in motion }\end{array}$ \\
\hline startY & $\begin{array}{l}\text { Location in the canvas; vertical starting position } \\
\text { of the object in motion }\end{array}$ \\
\hline endX & Horizontal ending position of the object in motion \\
\hline endY & Vertical ending position of the object in motion \\
\hline horiz_pix_frame & $\begin{array}{l}\text { Horizontal pixels by which the object moves } \\
\text { per frame }\end{array}$ \\
\hline horiz_pix_sec & $\begin{array}{l}\text { Horizontal pixels by which the object moves } \\
\text { per second }\end{array}$ \\
\hline vert_pix_frame & $\begin{array}{l}\text { Vertical pixels by which the object moves } \\
\text { per frame }\end{array}$ \\
\hline vert_pix_sec & $\begin{array}{l}\text { Vertical pixels by which the object moves } \\
\text { per second }\end{array}$ \\
\hline show_start_time & $\begin{array}{l}\text { Time to start presenting the object from the } \\
\text { beginning of the trial }\end{array}$ \\
\hline show_end_time & $\begin{array}{l}\text { Time to end presenting the object from the } \\
\text { beginning of the trial }\end{array}$ \\
\hline motion_start_time & $\begin{array}{l}\text { Time to start moving the object from the } \\
\text { beginning of the trial }\end{array}$ \\
\hline motion_end_time & $\begin{array}{l}\text { Time to end moving the object from the } \\
\text { beginning of the trial }\end{array}$ \\
\hline scale & Image scaling \\
\hline
\end{tabular}

(2015) evaluated the display durations of a white square presented in the program with Adobe Flash and JavaScript (HTML5). They showed that the durations were about $25 \mathrm{~ms}$ longer than the intended times, while within-machine variability was relatively small (generally less than $10 \mathrm{~ms}$ ). The present study investigated whether the program with the jspsych-psychophysics plugin showed the same (or better) accuracy as that reported by Reimers and Stewart. In addition, this study focused on not only the display duration but also the SOAs between visual stimuli, which have not been adequately evaluated.

\section{Method}

\section{Hardware and software}

Measurements were conducted using two Windows computers and two Macintosh computers, whose specifications are shown in Table 2. No external monitor was attached to any of the computers.

Google Chrome (75.03770) and Mozilla Firefox (68.0) were used in all computers. Microsoft Edge (42.17134) was used in the HP ProBook and Dell OptiPlex. There were two versions of Safari; v.11.1.2 was used in the MacBook Pro and v.12.1.1 was used in the iMac. Note that 32-bit Web browsers were used only in the Dell OptiPlex, and 64-bit Web browsers were used in the other computers.

\section{Procedures}

One program was coded using the jspsych-psychophysics plugin, and the other program was coded without the plugin. In both the programs, two white squares $(100 \times 100$ pixels $)$ were presented asynchronously on a black screen at different coordinates; one square was presented before and for a longer duration than the other. When the square was presented for $150 \mathrm{~ms}$, the other square was presented for $20 \mathrm{~ms}$ with SOA of $20,50,150$, and $500 \mathrm{~ms}$. Similarly, when the square was presented for $500 \mathrm{~ms}$, the other square was presented for $50 \mathrm{~ms}$ with the same SOAs. These display durations were identical to those in Reimers and Stewart's (2015) study except for the 20-ms duration condition, which was adopted to test how briefly a stimulus could be presented in a Web-based experiment. Note that a general display at $60 \mathrm{~Hz}$ cannot present a stimulus for $20 \mathrm{~ms}$ because the duration of one frame is $16.7 \mathrm{~ms}$. When the time of $20 \mathrm{~ms}$ was specified as a parameter of each program, it was expected that the stimulus would be presented for one or two frames. The purpose of this study was to compare the program using a new plugin with that without the plugin when the same timing parameter was given. Throughout this article, "intended" time refers to the time specified as a parameter in the programs. 
Table 2 List of machines tested

\begin{tabular}{|c|c|c|c|c|c|}
\hline Model name & OS & CPU/Bits & Memory size & Video card & Sound card/Speaker \\
\hline HP ProBook 430 G3 & $\begin{array}{l}\text { Windows } 10 \\
\text { pro } 1803\end{array}$ & Intel囚 Core ${ }^{\mathrm{TM}} \mathrm{i} 5-6200 \mathrm{U} / 64$ bits & $8 \mathrm{~GB}$ & $\begin{array}{l}\text { Intel® HD } \\
\text { Graphics } 520\end{array}$ & $\begin{array}{l}\text { Conexant ISST Audio } \\
\text { (Internal speaker) }\end{array}$ \\
\hline Dell OptiPlex 9010 & $\begin{array}{l}\text { Windows } 10 \\
\text { Education } 1803\end{array}$ & Intel囚 Core ${ }^{\mathrm{TM}} \mathrm{i} 7-3770 \mathrm{~S} / 32$ bits & $4 \mathrm{~GB}$ & $\begin{array}{l}\text { Intel® HD } \\
\text { Graphics } 4000\end{array}$ & $\begin{array}{l}\text { Realtek High Definition } \\
\quad \text { Audio (Internal speaker) }\end{array}$ \\
\hline $\begin{array}{l}\text { Apple MacBook Pro } \\
\text { (13-inch, late 2011) }\end{array}$ & $\begin{array}{l}\text { OS X El Capitan } \\
\text { 10.11.6 }\end{array}$ & Intel® Core $^{\mathrm{TM}} \mathrm{i} 5 / 64$ bits & $4 \mathrm{~GB}$ & $\begin{array}{l}\text { Intel® HD } \\
\text { Graphics } 3000\end{array}$ & $\begin{array}{l}\text { Dell Multimedia } \\
\text { speaker A215 }\end{array}$ \\
\hline $\begin{array}{l}\text { Apple iMac } \\
\text { (27-inch, late 2015) }\end{array}$ & $\begin{array}{l}\text { macOS Mojave } \\
10.14 .5\end{array}$ & Intel ${ }^{\circledR}$ Core $^{\mathrm{TM}} \mathrm{i} 5 / 64$ bits & $16 \mathrm{~GB}$ & $\begin{array}{l}\text { AMD Radeon } \\
\text { R9 M390 }\end{array}$ & Internal speaker \\
\hline
\end{tabular}

The stimuli were presented 100 times in each of the eight conditions except for the Dell OptiPlex, because the test program in this machine with the jspsych-psychophysics plugin with Google Chrome hung up. Although the reason was not certain, it was likely related to the browser version. Another five 32-bit computers were also tested, which revealed that the test programs using 32-bit Chrome hung up after some trials (mean $=637$ trials, standard deviation $[S D]=37.7$ ). Because of this problem, the number of repetitions of the test program with the new plugin on 32-bit Chrome in the OptiPlex was reduced to $50^{1}$.

All the programs were located on a Web server (see https:// jspsychophysics.hes.kyushu-u.ac.jp), and an Internet connection was needed to make them run. Other applications were not run during the measurements.

\section{Results and discussion}

There were some cases in which the display duration of a white square to be presented for $20 \mathrm{~ms}$ could not be captured properly by the photo-sensors attached to the Black Box. Table 3 shows the number of missing measurements during 100 trials except for the jspsych-psychophysics plugin condition with 32-bit Chrome in the OptiPlex (50 trials). This suggests that the square could not be presented for a very short display duration in rare cases, that is, the display frame dropped. There was no systematic bias with respect to the missing measurements among the SOAs, browser types, plugins, or computers. There were no missing measurements in the other duration conditions. In addition, for the no-plugin condition with Firefox in the OptiPlex, there was a trial where an exceedingly long display duration of $2089.5 \mathrm{~ms}$ was measured, whereas the intended duration was $20 \mathrm{~ms}$. The mean deviation from the intended display duration including and excluding the trial was $23.2 \mathrm{~ms}(\mathrm{SD}=103.2)$ and $18.0 \mathrm{~ms}$ $(\mathrm{SD}=11.7)$, respectively. Such extreme values were not observed in the other conditions.

\footnotetext{
${ }^{1}$ The issue related to the 32-bit Chrome seemed to be resolved when the browser was updated (at least in the Chrome v. 83.0.4102.61).
}

The measured display durations were more accurate and stable in the plugin conditions than in the no-plugin conditions. Table 4 shows the means and SDs of deviations from the intended display durations of the square calculated over different SOAs. The notable results are that (i) the large mean deviations (greater than $10 \mathrm{~ms}$ ) in the no-plugin conditions were reduced in the plugin conditions, and (ii) the SDs in the plugin conditions were smaller than or almost the same as those in the no-plugin conditions. These results show that the jspsych-psychophysics plugin improved the accuracy and precision of the display duration of visual stimuli. This improvement can be seen more clearly in the histogram of the actual display duration in each condition. The histograms in the no-plugin condition show multimodal distributions, with an interval of one frame. On the other hand, the histograms in the plugin condition show unimodal distributions, especially in Windows. All histograms related to Table 4 are available at Open Science Framework (https://osf.io/pj4sb/wiki/home/). In addition, in the no-plugin condition with Windows, Chrome presented the stimuli more accurately than Firefox, as also reported by Pronk et al. (2019). However, the marked difference among the browsers, including Edge and Safari, was not observed in the plugin condition. The small variability across browsers is ideal for a tool for online studies because it means participants can select any type of browser. It should be noted that the mean deviations in almost all the plugin conditions were negative, and this result is not consistent with that of the no-plugin conditions or with Reimers and Stewart's (2015) study. This inconsistency seems to be related to the time-specific method: in previous research, the jQuery's .show, .delay, and hide methods were used, whereas in the no-plugin conditions in this study the setTimeout method was used, and in the jspsych-psychophysics-plugin conditions the requestAnimationFrame method was used, by which stimulus presentation was in sync with the refresh rate of the display.

The measured SOAs were more accurate and stable in the plugin conditions than in the no-plugin conditions. Tables 5 and 6 show the mean deviations from the intended SOAs of the two squares for the two conditions, respectively: (a) one square was presented for $150 \mathrm{~ms}$ before the other was presented for $20 \mathrm{~ms}$, and (b) one square was presented for $500 \mathrm{~ms}$ 
Table 3 Number of missing measurements in the 20-ms display duration condition

\begin{tabular}{|c|c|c|c|c|c|c|}
\hline & \multicolumn{3}{|c|}{ No Plugin } & \multicolumn{3}{|l|}{ Plugin } \\
\hline & Chrome & Firefox & $\begin{array}{l}\text { Edge/ } \\
\text { Safari }\end{array}$ & Chrome & Firefox & $\begin{array}{l}\text { Edge/ } \\
\text { Safari }\end{array}$ \\
\hline ProBook & 0 & 0 & 0 & 0 & 1 & 2 \\
\hline OptiPlex & 7 & 0 & 0 & 0 & 1 & 0 \\
\hline MacBook Pro & 2 & 6 & 0 & 0 & 0 & 8 \\
\hline iMac & 0 & 7 & 0 & 8 & 0 & 0 \\
\hline
\end{tabular}

before the other was presented for $50 \mathrm{~ms}$. It is clearly shown in both Tables 5 and 6 that the large deviations (greater than 10 $\mathrm{ms}$ ) in the no-plugin conditions were reduced in the plugin conditions in almost all cases. However, it is noteworthy that even in the plugin conditions, the mean deviations were relatively large for the 20-ms SOA on all the browsers in the MacBook Pro and iMac, suggesting that the SOA of $20 \mathrm{~ms}$ was too short to adopt in a Web-based psychophysical experiment. This result might be related to the failure to replicate the priming effect (Crump et al., 2013; Semmelmann \& Weigelt, 2017). Except for the 20-ms condition, the jspsychpsychophysics plugin worked properly, and improved the performance of the jsPsych program. All histograms related to
Tables 5 and 6 are available at Open Science Framework (https://osf.io/pj4sb/wiki/home/).

\section{Study 2: Evaluation of the SOAs between visual and auditory stimuli}

The jspsych-psychophysics plugin presents audio stimuli using the same method as the jsPsych (de Leeuw, 2015) provides, that is, using the Web Audio API, which is a multifunctional library for playing audio on the Web. Reimers and Stewart (2016) reported that although the auditory duration was relatively accurate, the SOAs between visual and auditory

Table 4 Means and SDs of deviations from intended display durations of the square

\begin{tabular}{|c|c|c|c|c|c|c|}
\hline & \multicolumn{3}{|l|}{ No Plugin } & \multicolumn{3}{|l|}{ Plugin } \\
\hline & Chrome & Firefox & Edge/Safari & Chrome & Firefox & Edge/Safari \\
\hline \multicolumn{7}{|l|}{ ProBook } \\
\hline 20-ms display duration & $-5.6(8.8)$ & $10.4(11.3)$ & $11.2(11.4)$ & $-13.3(0.3)$ & $-10.9(1.9)$ & $-13.6(3.1)$ \\
\hline 50-ms display duration & $-3.2(8.5)$ & $10.8(12.2)$ & $5.1(15.7)$ & $-9.2(0.3)$ & $-7.2(1.7)$ & $-9.8(4.1)$ \\
\hline 150-ms display duration & $-2.4(8.3)$ & $29.8(18.0)$ & $28.3(28.2)$ & $-11.0(2.7)$ & $-8.2(2.5)$ & $-8.0(2.6)$ \\
\hline 500-ms display duration & $2.3(10.9)$ & $15.1(14.9)$ & $30.5(23.1)$ & $-10.7(3.1)$ & $-8.7(1.7)$ & $-8.3(4.2)$ \\
\hline \multicolumn{7}{|l|}{ OptiPlex } \\
\hline 20-ms display duration & $-5.9(6.8)$ & $18.0(11.7)$ & $20.4(8.1)$ & $-8.2(0.2)$ & $-8.2(4.2)$ & $-8.3(1.5)$ \\
\hline 50-ms display duration & $4.6(13.3)$ & $10.2(11.5)$ & $14.7(13.2)$ & $-4.6(0.1)$ & $-4.9(0.1)$ & $-4.7(3.1)$ \\
\hline 150-ms display duration & $10.7(8.4)$ & $41.7(20.7)$ & $28.6(26.6)$ & $-4.4(1.2)$ & $-3.9(14.5)$ & $-4.7(2.4)$ \\
\hline 500-ms display duration & $13.2(16.3)$ & $31.0(13.7)$ & $30.9(16.4)$ & $-5.2(0.1)$ & $-6.1(1.9)$ & $-5.2(3.3)$ \\
\hline \multicolumn{7}{|l|}{ MacBook Pro } \\
\hline 20-ms display duration & $-7.7(8.8)$ & $-9.0(10.3)$ & $-6.3(8.6)$ & $-1.4(7.8)$ & $-0.8(8.1)$ & $-0.7(9.6)$ \\
\hline 50-ms display duration & $-2.6(8.5)$ & $-9.4(10.6)$ & $-4.4(7.1)$ & $-9.9(0.7)$ & $-8.5(4.6)$ & $-6.1(7.3)$ \\
\hline 150-ms display duration & $0.6(8.2)$ & $4.2(9.9)$ & $-0.3(8.1)$ & $-6.5(0.2)$ & $-7.6(7.6)$ & $-7.5(10.4)$ \\
\hline 500-ms display duration & $6.8(9.4)$ & $7.9(13.8)$ & $3.5(8.4)$ & $-5.7(0.2)$ & $-8.0(5.6)$ & $-11.3(9.5)$ \\
\hline \multicolumn{7}{|l|}{ iMac } \\
\hline 20-ms display duration & $-0.3(11.1)$ & $1.0(9.4)$ & $-2.4(7.5)$ & $6.3(7.1)$ & $3.6(8.1)$ & $4.5(7.9)$ \\
\hline 50-ms display duration & $2.8(8.2)$ & $0.8(7.4)$ & $-0.9(5.9)$ & $-3.2(1.2)$ & $-4.0(7.4)$ & $4.7(8.3)$ \\
\hline 150-ms display duration & $5.6(8.3)$ & $13.6(7.2)$ & $0.6(6.8)$ & $-2.2(4.6)$ & $-3.3(8.5)$ & $7.1(8.5)$ \\
\hline 500-ms display duration & $12.6(11.0)$ & $14.9(10.6)$ & $2.5(7.8)$ & $-2.4(3.1)$ & $-3.6(9.0)$ & $8.2(8.4)$ \\
\hline
\end{tabular}

Note. Times are given in milliseconds. SDs are presented in brackets. Positive mean indicates that the actual display duration was longer than the intended duration 
Table 5 Means and SDs of deviations from intended SOAs between the onset of the 150-ms square and the 20-ms square

\begin{tabular}{|c|c|c|c|c|c|c|}
\hline & \multicolumn{3}{|l|}{ No Plugin } & \multicolumn{3}{|l|}{ Plugin } \\
\hline & Chrome & Firefox & Edge/Safari & Chrome & Firefox & Edge/Safari \\
\hline \multicolumn{7}{|l|}{ ProBook } \\
\hline 20-ms SOA & $9.3(8.3)$ & $32.5(8.1)$ & $28.5(13.2)$ & $1.3(2.9)$ & $2.2(2.9)$ & $5.0(2.4)$ \\
\hline 50-ms SOA & $9.0(7.8)$ & $35.0(9.5)$ & $19.9(15.5)$ & $4.4(1.7)$ & $4.8(2.9)$ & $8.1(3.4)$ \\
\hline 150-ms SOA & $9.2(7.5)$ & $31.0(8.6)$ & $22.3(15.5)$ & $4.5(2.4)$ & $4.8(2.3)$ & $7.9(3.0)$ \\
\hline 500-ms SOA & $12.5(8.7)$ & $21.1(10.2)$ & $42.3(16.6)$ & $4.8(3.3)$ & $4.5(2.4)$ & $7.6(2.9)$ \\
\hline \multicolumn{7}{|l|}{ OptiPlex } \\
\hline 20-ms SOA & $10.5(9.4)$ & $40.3(11.0)$ & $29.0(7.8)$ & $1.8(0.2)$ & $0.9(0.1)$ & $1.3(0.1)$ \\
\hline 50-ms SOA & $7.5(10.8)$ & $32.5(9.3)$ & $21.4(5.8)$ & $4.9(0.1)$ & $4.1(0.1)$ & $5.2(4.0)$ \\
\hline 150-ms SOA & $22.4(8.3)$ & $31.2(8.3)$ & $10.3(10.2)$ & $4.6(0.1)$ & $3.9(0.1)$ & $4.1(2.9)$ \\
\hline 500-ms SOA & $23.9(9.3)$ & $29.4(10.1)$ & $23.9(7.9)$ & $3.7(0.1)$ & $2.9(0.1)$ & $3.3(0.1)$ \\
\hline \multicolumn{7}{|l|}{ MacBook Pro } \\
\hline 20-ms SOA & $11.2(8.1)$ & $12.5(8.7)$ & $9.5(7.9)$ & $22.1(0.5)$ & $19.2(5.0)$ & $11.3(9.3)$ \\
\hline 50-ms SOA & $16.3(8.4)$ & $14.5(8.0)$ & $10.6(6.7)$ & $8.8(0.5)$ & $7.5(6.7)$ & $3.2(8.3)$ \\
\hline $150-\mathrm{ms} \mathrm{SOA}$ & $13.2(7.5)$ & $20.8(10.0)$ & $10.3(6.4)$ & $9.1(0.5)$ & $5.9(7.8)$ & $2.1(9.1)$ \\
\hline 500-ms SOA & $21.1(7.9)$ & $14.4(14.7)$ & $17.2(8.3)$ & $9.8(0.6)$ & $5.3(6.6)$ & $2.6(9.5)$ \\
\hline \multicolumn{7}{|l|}{ iMac } \\
\hline 20-ms SOA & $13.7(13.1)$ & $9.3(8.3)$ & $5.5(7.7)$ & $16.9(5.5)$ & $13.7(6.7)$ & $15.1(5.3)$ \\
\hline 50-ms SOA & $8.6(7.6)$ & $12.6(8.7)$ & $5.8(5.6)$ & $4.1(2.4)$ & $4.0(7.9)$ & $3.1(7.6)$ \\
\hline 150-ms SOA & $10.1(8.1)$ & $18.5(5.9)$ & $6.2(5.8)$ & $4.2(2.4)$ & $2.1(9.8)$ & $3.0(7.7)$ \\
\hline 500-ms SOA & $15.1(8.2)$ & $6.1(7.3)$ & $7.7(7.1)$ & $4.1(4.1)$ & $3.2(8.5)$ & $2.5(6.6)$ \\
\hline
\end{tabular}

Note. Times are given in milliseconds. SDs are presented in brackets. Positive mean indicates that the actual SOA was longer than the intended SOA

stimuli varied substantially across browser-computer combinations (see Slote and Strand, 2016 for Web-based cognitive psychological research with auditory stimuli). The present study investigated whether the program with the jspsychpsychophysics plugin showed the same (or better) accuracy as that reported by Reimers and Stewart.

\section{Methods}

\section{Hardware and software}

Measurements were conducted using computers identical to those used in Study 1, whose specifications are shown in Table 2. An external speaker was attached to the MacBook Pro because the volume from the internal speaker was too low to measure. The versions of the Web browser were also identical to those used in Study 1.

\section{Procedures}

One program was coded using the jspsych-psychophysics plugin, and the other program was coded without the plugin. In the jspsych-psychophysics plugin condition, a white square $(100 \times 100$ pixels $)$ was presented for $500 \mathrm{~ms}$ on a black screen, ahead of or at the same time as a sine-wave sound of $440 \mathrm{~Hz}$ (100-ms duration). The SOAs between the onset of the square and that of the sound were $0,20,50,150$, and $500 \mathrm{~ms}$. In each of the five conditions, the stimuli were presented 100 times.

In the no-plugin condition, a white square was presented for $500 \mathrm{~ms}$ on a black screen, and this was followed by the presentation of the sine-wave sound of $440 \mathrm{~Hz}$ for $100 \mathrm{~ms}$. Because there was no simple way to present an auditory stimulus during presentation of visual stimuli using only the core methods of the jsPsych, the sound was presented immediately after the disappearance of the square; in other words, there was only a 500-ms SOA condition. However, a reviewer suggested a sophisticated way to present an image and an audio simultaneously, and the stimulus presentation in accordance with the comment is identical to that adopted by Bridges, Pitiot, MacAskill, and Peirce (2020). Using this method, the 0-ms SOA data in the no-plugin condition were recorded post hoc in the ProBook, OptiPlex, and iMac. Note also that the Web Audio API was turned off in the original (the 500-ms SOA) condition, and was turned on in the 0 -ms SOA condition. Both the visual and audio stimuli were presented 100 times.

All the programs were located on a Web server (see https:// jspsychophysics.hes.kyushu-u.ac.jp), and an Internet 
Table 6 Means and SDs of deviations from intended SOAs between the onset of the 500-ms square and the 50-ms square

\begin{tabular}{|c|c|c|c|c|c|c|}
\hline & \multicolumn{3}{|l|}{ No Plugin } & \multicolumn{3}{|l|}{ Plugin } \\
\hline & Chrome & Firefox & Edge/Safari & Chrome & Firefox & Edge/Safari \\
\hline \multicolumn{7}{|l|}{ ProBook } \\
\hline 20-ms SOA & $10.3(8.7)$ & $36.3(5.7)$ & $21.7(13.7)$ & $1.8(2.4)$ & $1.6(0.2)$ & $5.3(2.9)$ \\
\hline 50-ms SOA & $11.4(8.3)$ & $37.6(6.4)$ & $20.1(12.4)$ & $5.1(2.9)$ & $4.7(2.9)$ & $7.7(3.3)$ \\
\hline 150-ms SOA & $9.8(8.2)$ & $35.3(7.3)$ & $21.6(12.8)$ & $4.5(1.7)$ & $4.8(0.2)$ & $8.0(4.8)$ \\
\hline 500-ms SOA & $10.0(7.9)$ & $8.5(8.3)$ & $24.6(14.9)$ & $4.9(4.6)$ & $4.4(1.7)$ & $7.9(4.1)$ \\
\hline \multicolumn{7}{|l|}{ OptiPlex } \\
\hline 20-ms SOA & $9.4(9.0)$ & $41.4(9.3)$ & $29.0(7.9)$ & $1.7(0.1)$ & $0.9(0.1)$ & $1.3(0.1)$ \\
\hline 50-ms SOA & $4.5(9.2)$ & $31.7(8.6)$ & $22.3(4.9)$ & $4.9(0.1)$ & $4.1(0.1)$ & $4.7(3.7)$ \\
\hline 150-ms SOA & $21.4(11.2)$ & $33.3(9.1)$ & $18.1(9.1)$ & $4.7(0.1)$ & $3.9(0.1)$ & $4.2(5.3)$ \\
\hline 500-ms SOA & $7.5(10.3)$ & $22.5(8.0)$ & $21.2(5.1)$ & $3.7(0.1)$ & $2.6(3.3)$ & $4.1(3.6)$ \\
\hline \multicolumn{7}{|l|}{ MacBook Pro } \\
\hline 20-ms SOA & $9.7(7.5)$ & $14.1(11.3)$ & $11.0(8.4)$ & $22.1(0.5)$ & $19.2(5.0)$ & $12.3(10.5)$ \\
\hline 50-ms SOA & $14.1(8.0)$ & $22.5(14.1)$ & $11.9(7.5)$ & $8.9(0.6)$ & $6.6(7.1)$ & $3.2(8.6)$ \\
\hline $150-\mathrm{ms} \mathrm{SOA}$ & $16.3(8.4)$ & $21.6(13.3)$ & $10.5(6.4)$ & $9.0(0.6)$ & $6.7(6.2)$ & $1.4(10.2)$ \\
\hline 500-ms SOA & $13.8(7.4)$ & $31.1(13.4)$ & $13.1(7.6)$ & $9.7(0.6)$ & $6.1(5.8)$ & $2.5(8.5)$ \\
\hline \multicolumn{7}{|l|}{ iMac } \\
\hline 20-ms SOA & $11.1(11.3)$ & $21.8(7.8)$ & $4.8(7.5)$ & $16.9(1.7)$ & $13.5(6.9)$ & $16.1(3.7)$ \\
\hline 50-ms SOA & $11.2(8.3)$ & $20.6(10.0)$ & $5.3(5.1)$ & $3.7(1.7)$ & $2.0(10.1)$ & $3.5(3.8)$ \\
\hline 150-ms SOA & $11.8(8.3)$ & $17.0(11.4)$ & $6.2(6.2)$ & $3.9(2.4)$ & $3.5(8.5)$ & $4.1(4.0)$ \\
\hline $500-\mathrm{ms} \mathrm{SOA}$ & $12.8(9.0)$ & $19.5(12.8)$ & $7.1(6.6)$ & $3.4(2.9)$ & $3.1(8.4)$ & $4.1(3.0)$ \\
\hline
\end{tabular}

Note. Times are given in milliseconds. SDs are presented in brackets. Positive mean indicates that the actual SOA was longer than the intended SOA

connection was needed to make them run. Other applications were not run during the measurements.

\section{Results and discussion}

There were some cases in which the sound could not be properly captured by the microphones attached to the Black Box. For the no-plugin condition with Edge in the ProBook, the program could not play back the sound stably. The reason was unknown, and the problem did not depend on the use of the internal or external speakers. Note that the jspsychpsychophysics program could play back the sound under the same settings. In addition, for the no-plugin condition with Firefox in the MacBook Pro, the sound was recorded as divided into three short parts. These data were eliminated, leaving a total of 97 trials in this condition.

The measured sound durations were more accurate and stable in the plugin conditions than in the no-plugin conditions. Table 7 shows the means and SDs of deviations from the intended display and sound durations. Because only the 500-ms SOA was used for the no-plugin condition, the means and SDs for the plugin condition were also calculated using the data for the 500-ms SOA. For both the no-plugin and plugin conditions, the visual and auditory stimuli were generally presented accurately and precisely. More importantly, the large means and SDs of the sound duration in the noplugin conditions with Safari in the MacBook Pro and iMac were reduced in the plugin conditions. This result indicates that the jspsych-psychophysics plugin reduces variability between browsers and computers.

The measured SOAs between the onset of the square and that of the sound were inaccurate in both the plugin and noplugin conditions. As shown in Table 8, the means of deviations from the intended SOAs, including the data recorded post hoc, were substantial (from about $5 \mathrm{~ms}$ to $50 \mathrm{~ms}$ ), and were variable across browser-computer combinations. This result is consistent with the reports by Reimers and Stewart (2016) and Bridges et al. (2020). However, this does not mean that visual and auditory interaction cannot be investigated in online studies. There are some alternative ways. First, it is possible that a movie file can be used to present visual and audio stimuli, as Reimers and Stewart suggested. Second, given that Bazilinskyy and de Winter (2018) were able to successfully replicate the effect of the SOA on reaction times, a within-participant experimental design is available. Lastly, although it takes additional time, the difference between the onset of a visual stimulus and that of an auditory stimulus can be determined by adopting a staircase procedure (Woods 
Table 7 Means and SDs of deviations from intended display and sound durations

\begin{tabular}{|c|c|c|c|c|c|c|}
\hline & \multicolumn{3}{|l|}{ No Plugin } & \multicolumn{3}{|l|}{ Plugin } \\
\hline & Chrome & Firefox & Edge/Safari & Chrome & Firefox & Edge/Safari \\
\hline \multicolumn{7}{|l|}{ ProBook } \\
\hline 500-ms display duration & $-3.2(8.3)$ & $1.0(12.6)$ & - & $-6.9(2.3)$ & $-8.4(1.7)$ & $-7.9(3.7)$ \\
\hline $100-\mathrm{ms}$ sound duration & $-1.2(0.7)$ & $-2.9(0.3)$ & - & $-1.1(0.7)$ & $-1.7(0.5)$ & $-1.5(1.2)$ \\
\hline \multicolumn{7}{|l|}{ OptiPlex } \\
\hline 500-ms display duration & $12.9(8.5)$ & $13.9(17.9)$ & $11.5(9.0)$ & $-5.5(0.2)$ & $-5.6(0.2)$ & $-5.8(1.7)$ \\
\hline 100 -ms sound duration & $3.8(0.8)$ & $-1.2(0.2)$ & $-4.5(3.7)$ & $3.3(1.4)$ & $3.7(1.1)$ & $3.5(0.9)$ \\
\hline \multicolumn{7}{|l|}{ MacBook Pro } \\
\hline 500-ms display duration & $-3.7(6.8)$ & $-1.5(7.6)$ & $-6.0(7.1)$ & $-6.6(0.4)$ & $-8.0(5.0)$ & $-7.7(9.2)$ \\
\hline 100 -ms sound duration & $-1.1(0.4)$ & $-5.7(3.0)$ & $-16.4(2.1)$ & $-1.1(0.5)$ & $-1.0(0.3)$ & $-0.5(0.3)$ \\
\hline \multicolumn{7}{|l|}{ iMac } \\
\hline 500-ms display duration & $1.2(7.6)$ & $2.5(6.9)$ & $-0.4(6.6)$ & $-2.8(5.3)$ & $-0.9(9.2)$ & $5.4(9.9)$ \\
\hline $100-\mathrm{ms}$ sound duration & $-4.2(0.5)$ & $-4.9(0.5)$ & $-21.7(2.0)$ & $-4.1(0.5)$ & $-1.6(0.7)$ & $-4.2(0.3)$ \\
\hline
\end{tabular}

Note. Times are given in milliseconds. SDs are presented in brackets. Positive mean indicates that the actual display and sound duration was longer than the intended duration. A dash indicates that data were not obtained

et al., 2015). More importantly, although the new plugin could not present the visual and audio stimuli accurately, the means of the deviations were lower than those for the no-plugin conditions, especially for Chrome in Windows and Safari in Mac.
In that sense, the jspsych-psychophysics plugin improved the performance of the jsPsych program. All histograms related to Tables 7 and 8 are available at Open Science Framework (https://osf.io/pj4sb/wiki/home/).

Table 8 Means and SDs of deviations from intended SOAs between square and sound

\begin{tabular}{|c|c|c|c|c|c|c|}
\hline & \multicolumn{3}{|l|}{ No Plugin } & \multicolumn{3}{|l|}{ Plugin } \\
\hline & Chrome & Firefox & Edge/Safari & Chrome & Firefox & Edge/Safari \\
\hline \multicolumn{7}{|l|}{ ProBook } \\
\hline 0 -ms SOA & $33.2(10.7)$ & $15.8(7.7)$ & $4.2(8.6)$ & $-2.9(6.7)$ & $-8.8(7.4)$ & $-9.9(5.2)$ \\
\hline 20-ms SOA & - & - & - & $-4.7(6.1)$ & $-8.6(9.1)$ & $-13.2(4.1)$ \\
\hline 50-ms SOA & - & - & - & $-4.7(6.6)$ & $-10.3(9.0)$ & $-11.9(4.3)$ \\
\hline 150-ms SOA & - & - & - & $-4.2(5.8)$ & $-5.3(7.4)$ & $-11.4(4.4)$ \\
\hline 500-ms SOA & $40.8(10.3)$ & $0.2(9.0)$ & - & $-4.9(6.4)$ & $-4.7(7.9)$ & $-11.6(4.1)$ \\
\hline \multicolumn{7}{|l|}{ OptiPlex } \\
\hline 0 -ms SOA & $-6.6(9.2)$ & $-25.1(7.3)$ & $-36.5(9.4)$ & $-18.8(6.2)$ & $-17.0(6.5)$ & $-25.2(4.5)$ \\
\hline 20-ms SOA & - & - & - & $-18.0(5.7)$ & $-17.5(6.4)$ & $-27.8(3.1)$ \\
\hline 50-ms SOA & - & - & - & $-18.4(5.8)$ & $-24.2(5.7)$ & $-25.0(3.3)$ \\
\hline 150-ms SOA & - & - & - & $-17.2(7.2)$ & $-24.1(6.3)$ & $-24.7(4.2)$ \\
\hline 500-ms SOA & $40.7(8.4)$ & $8.6(17.2)$ & $-33.8(8.5)$ & $-17.9(5.3)$ & $-18.4(6.3)$ & $-24.8(3.6)$ \\
\hline \multicolumn{7}{|l|}{ MacBook Pro } \\
\hline 0 -ms SOA & - & - & - & $-33.9(4.8)$ & $-29.2(7.4)$ & $-26.1(8.7)$ \\
\hline 20-ms SOA & - & - & - & $-33.8(4.0)$ & $-30.4(8.3)$ & $-25.7(7.8)$ \\
\hline 50-ms SOA & - & - & - & $-33.3(4.6)$ & $-30.4(8.9)$ & $-25.5(8.5)$ \\
\hline $150-\mathrm{ms} \mathrm{SOA}$ & - & - & - & $-33.8(4.3)$ & $-29.4(8.4)$ & $-25.2(8.6)$ \\
\hline 500-ms SOA & $8.1(6.2)$ & $-28.1(5.4)$ & $118.0(9.1)$ & $-34.5(4.9)$ & $-30.0(7.1)$ & $-27.4(9.5)$ \\
\hline \multicolumn{7}{|l|}{ iMac } \\
\hline 0 -ms SOA & $-6.0(5.6)$ & $-36.8(5.6)$ & $-25.9(5.4)$ & $-13.2(7.1)$ & $-30.3(9.8)$ & $-24.1(8.8)$ \\
\hline 20-ms SOA & - & - & - & $-15.7(8.4)$ & $-48.5(10.6)$ & $-20.1(5.7)$ \\
\hline 50-ms SOA & - & - & - & $-14.9(7.1)$ & $-48.0(9.9)$ & $-22.4(9.3)$ \\
\hline 150-ms SOA & - & - & - & $-13.4(6.1)$ & $-42.3(13.3)$ & $-20.8(7.2)$ \\
\hline 500-ms SOA & $36.4(5.1)$ & $-33.0(6.1)$ & $141.8(6.5)$ & $-14.2(6.9)$ & $-42.8(11.2)$ & $-22.4(8.9)$ \\
\hline
\end{tabular}

Note. Times are given in milliseconds. SDs are presented in brackets. Positive mean indicates that the actual SOA was longer than the intended SOA. A dash indicates that data were not obtained. The data for the 0-ms SOA condition in the no-plugin condition were recorded post hoc in accordance with a reviewer's comment 


\section{Conclusion}

This study introduced a new jsPsych plugin for conducting a Web-based psychophysical experiment, and evaluated both the stimulus duration and the SOAs, the latter of which have not been adequately evaluated previously. The new plugin reduces the variability in both the duration and SOA across browser-computer combinations, although the presentation of a sound file is somewhat limited. Moreover, after the submission of this paper, the plugin was updated to specify timing information not only in milliseconds but also in frames. The use of the plugin is recommended for any kind of experiments using jsPsych, because visual stimuli are presented in sync with the refresh rate of the display. In other words, the visual stimuli will be presented more accurately than when the plugin is not used.

Acknowledgements I thank Hiroyuki Mitsudo and Kentaro Yamamoto for their valuable comments on an earlier version of this manuscript.

Open practices statement The data and materials for all experiments are available at Open Science Framework (https://osf.io/pj4sb/), and none of the experiments was preregistered.

\section{References}

Barnhoorn, J. S., Haasnoot, E., Bocanegra, B. R., \& van Steenbergen, H. (2015). QRTEngine: An easy solution for running online reaction time experiments using Qualtrics. Behavior Research Methods, 47(4), 918-929. https://doi.org/10.3758/s13428-014-0530-7

Bazilinskyy, P., \& de Winter, J. C. F. (2018). Crowdsourced Measurement of Reaction Times to Audiovisual Stimuli With Various Degrees of Asynchrony. Human Factors, 60(8), 11921206. https://doi.org/10.1177/0018720818787126

Bridges, D., Pitiot, A., MacAskill, M. R., \& Peirce, J. W. (2020). The timing mega-study: comparing a range of experiment generators, both lab-based and online. PsyArXiv, 10.31234/osf.io/d6nu5

Chetverikov, A., \& Upravitelev, P. (2016). Online versus offline: The Web as a medium for response time data collection. Behavior Research Methods, 48(3), 1086-1099. https://doi.org/10.3758/ s13428-015-0632-x

Crump, M. J. C., McDonnell, J. V., \& Gureckis, T. M. (2013). Evaluating Amazon's Mechanical Turk as a Tool for Experimental Behavioral Research. PLoS ONE, 8(3). https://doi.org/10.1371/journal.pone. 0057410

de Leeuw, J. R. (2015). jsPsych: A JavaScript library for creating behavioral experiments in a Web browser. Behavior Research Methods, 47(1), 1-12. https://doi.org/10.3758/s13428-014-0458-y

de Leeuw, J. R., \& Motz, B. A. (2016). Psychophysics in a Web browser? Comparing response times collected with JavaScript and Psychophysics Toolbox in a visual search task. Behavior Research Methods, 48(1), 1-12. https://doi.org/10.3758/s13428-015-0567-2

Garaizar, P., \& Reips, U. D. (2019). Best practices: Two Web-browserbased methods for stimulus presentation in behavioral experiments with high-resolution timing requirements. Behavior Research Methods, 51(3), 1441-1453. https://doi.org/10.3758/s13428-018$1126-4$
Pauszek, J. R., Sztybel, P., \& Gibson, B. S. (2017). Evaluating Amazon's Mechanical Turk for psychological research on the symbolic control of attention. Behavior Research Methods, 49(6), 1969-1983. https:// doi.org/10.3758/s13428-016-0847-5

Pinet, S., Zielinski, C., Mathôt, S., Dufau, S., Alario, F.-X., \& Longcamp, M. (2017). Measuring sequences of keystrokes with jsPsych: Reliability of response times and interkeystroke intervals. Behavior Research Methods, 49(3), 1163-1176. https://doi.org/10. 3758/s13428-016-0776-3

Pronk, T., Wiers, R.W., Molenkamp, B. \& Murre, J. (2019). Mental chronometry in the pocket? Timing accuracy of web applications on touchscreen and keyboard devices Behavior Research Methods https://doi.org/10.3758/s13428-019-01321-2

Reimers, S., \& Stewart, N. (2015). Presentation and response timing accuracy in Adobe Flash and HTML5/JavaScript Web experiments. Behavior Research Methods, 47(2), 309-327. https://doi.org/10. 3758/s13428-014-0471-1

Reimers, S., \& Stewart, N. (2016). Auditory presentation and synchronization in Adobe Flash and HTML5/JavaScript Web experiments. Behavior Research Methods, 48(3), 897-908. https://doi.org/10. 3758/s13428-016-0758-5

Reips, U.-D., \& Neuhaus, C. (2002). WEXTOR: a Web-based tool for generating and visualizing experimental designs and procedures. Behavior Research Methods, Instruments, \& Computers : A Journal of the Psychonomic Society, Inc, 34(2), 234-240. https:// doi.org/10.3758/BF03195449

Richter, J., \& Gast, A. (2017). Distributed practice can boost evaluative conditioning by increasing memory for the stimulus pairs. Acta Psychologica, 179(April), 1-13. https://doi.org/10.1016/j.actpsy. 2017.06.007

Sasaki, K., \& Yamada, Y. (2019). Crowdsourcing visual perception experiments : a case of contrast threshold. https://doi.org/10.7717/ peerj. 8339

Schubert, T. W., Murteira, C., Collins, E. C., \& Lopes, D. (2013). ScriptingRT: A Software Library for Collecting Response Latencies in Online Studies of Cognition. PLOS ONE, 8(6). https:// doi.org/10.1371/journal.pone.0067769

Semmelmann, K., \& Weigelt, S. (2017). Online psychophysics: reaction time effects in cognitive experiments. Behavior Research Methods, 49(4), 1241-1260. https://doi.org/10.3758/s13428-016-0783-4

Slote, J., \& Strand, J. F. (2016). Conducting spoken word recognition research online: Validation and a new timing method. Behavior Research Methods, 48(2), 553-566. https://doi.org/10.3758/ s13428-015-0599-7

Stewart, N., Chandler, J., \& Paolacci, G. (2017). Crowdsourcing Samples in Cognitive Science. Trends in Cognitive Sciences, 21(10), 736748. https://doi.org/10.1016/j.tics.2017.06.007

van Steenbergen, H., Band, G. P. H., \& Hommel, B. (2015). Does conflict help or hurt cognitive control? Initial evidence for an inverted Ushape relationship between perceived task difficulty and conflict adaptation. Frontiers in Psychology, 6. https://doi.org/10.3389/ fpsyg.2015.00974

von Bastian, C. C., Locher, A., \& Ruflin, M. (2013). Tatool: A Javabased open-source programming framework for psychological studies. Behavior Research Methods, 45(1), 108-115. https://doi.org/10. 3758/s13428-012-0224-y

Woods, A. T., Velasco, C., Levitan, C. A., Wan, X., \& Spence, C. (2015). Conducting perception research over the internet: a tutorial review. PeerJ, 3, e1058. https://doi.org/10.7717/peerj.1058

Publisher's note Springer Nature remains neutral with regard to jurisdictional claims in published maps and institutional affiliations. 